\title{
A STUDY ON SELF-DRIVING CAR AN APPLICATION OF IoT
}

\author{
GEETIKA MATHUR ${ }^{1}$, HARSHIT SHARMA ${ }^{2} \&$ RISHABH PANDEY ${ }^{3}$ \\ ${ }^{1,2}$ Department of Computer Science Engineering, Arya Institute of Engineering \& Technology \\ Rajasthan Technical University, Jaipur, Rajasthan, India \\ ${ }^{3}$ Department of Mechanical Engineering, Arya Institute of Engineering \& Technology
}

Rajasthan Technical University, Jaipur, Rajasthan, India

\begin{abstract}
We are plunged into a new era of applied science i.e., IoT (Internet of Things). Fundamentally, the Internet of Things is concatenating insensate devices over the internet without human interference. As a result of IoT, a huge amount of data can be generated and stored. This data can be processed with a utilitarian approach that can "command and control" the things to make our lives trouble-free. The Internet of Things is a system which reticulates computing devices, digital and mechanical machines, objects, animals or people etc. Some applications of IoT are Self-Driving cars and home appliances that contain sensors, electronic devices and actuators which allow these things to connect, interact and exchange data. Self-Driving car requires an enormous quantity of data collection and processing. The information acquired by the electronic devices used in the self-driving car is about the road, actual path, traffic and how to navigate around any obstacle. This data is shared between IoT connected cars and is uploaded wirelessly to a cloud system to be analysed and put to use improving the automation. The aim of this paper is to provide an encyclopaedic analysis of IoT. It also describes the importance of IoT in Self-Driving car and its forthcoming challenges.

KEYWORDS: IoT \& Self-Driving Car
\end{abstract}

Received: Mar 01, 2019; Accepted: Mar 21, 2019; Published: Apr 16, 2019; Paper Id.: IJCNWMCJUN20194

\section{INTRODUCTION}

The main objective of IoT is to assist everyone with their day-to-day challenges which can be done by using various devices such as sensors, actuators, smart appliances etc. All the devices are interconnected to communicate with each other with the help of a network. The devices which were not electronically supported, have started embedding with sensors and microprocessor to interact and communicate with each other. Around 5\% of man-made objects currently have microprocessor embedded in them. The sensors like RFID (Radio frequency Identification), LIDAR (Light Detection and Ranging), wireless sensor, satellite positioning etc., acquires the basic information which helps in processing the data. Self-Driving Cars are the most vivid example of the IoT. SelfDriving cars are also known as robot car, driverless car or autonomous car, is a vehicle that is capable of sensing it's environment or moving with less or no human input. Autonomous cars combine a variety of sensors - radar, LIDAR, sonar, GPS, odometer, and inertial measurement units to capture their surroundings. This paper discusses the brief functioning of sensors used in autonomous cars. The future predictions of the self-driving car are also discussed in this paper with facts and figures. 


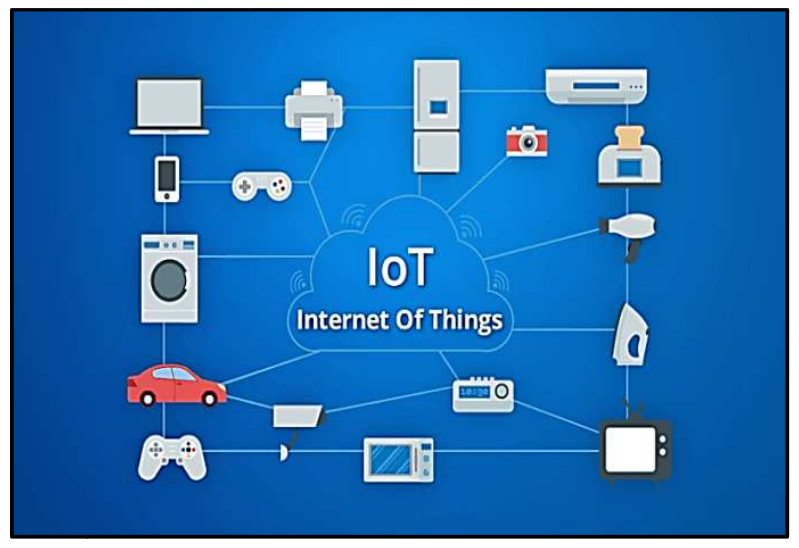

Figure 1: Internet of Things

\section{ARCHITECTURE OF IOT}

There is no single architectural design which is concurred universally because each user or company or organisation for that matter has a different requirement. Therefore, different researchers propound different architectures. The working of the network module is described in this architecture. The fundamental architecture of IOT is sliced into three layers Perception Layer, Network Layer and Application Layer.

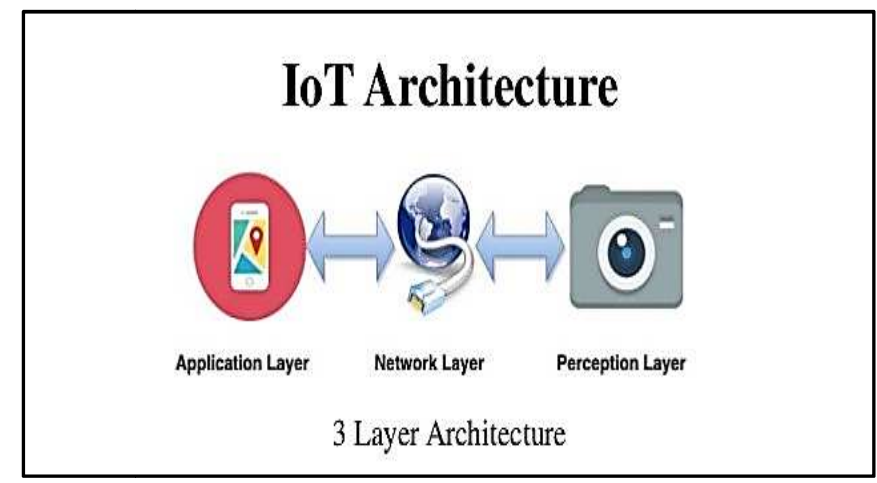

Figure 2: Layers of IOT

\section{Perception Layer}

The perception layer is the lowest layer of the standard architecture of IoT. The other name of perception layer is recognition layer. The main aim of perception layer is to amass useful information from surrounding (such as heterogeneous devices, sensors type, humidity and temperature etc.) and mutate them in a digital setup. The objective of this information collection is to identify unique address and to establish a communication network between technologies such as RFID, Bluetooth and Near-Field Communication (NFC).

\section{Network Layer}

This layer is considered as the brain of the fundamental layers of IoT. Assisting Secured data transmission between application and perception layer is accomplished by the network layer. This layer is used for establishing a link between internet and communication-based networks. Various researchers of communicational technologies, shows that network layer is the core layer of IoT 6 (i.e. competent in forwarding the information for executing procedures). This layer looks after routing abilities and unique addressing in order to uniquely integrate an uncountable number of devices into a single network. Technologies like wired, wireless and satellite etc. are used in this process. 


\section{Application Layer}

This layer is the topmost layer of the fundamental architecture of IoT. According to the distinctive prepossession, services are provided to the customers by this layer. The objective of this layer is to channelize the considerable gap between users and applications. This layer also links the industry to acquire a highly advance application for solutions such as health and disaster monitoring, medical and ecological environment, transportation and handling global management related to all applications.

\section{SELF-DRIVING CAR}

Self-Driving Cars are the future of transportation. Major Technology companies have spent billions of dollars developing self-driving cars. In the belief that the market for them one day be worth trillions of dollars. Self-Driving Cars would greatly reduce the price of transport of consumer since there is no human in the loop. To navigate efficiently, a selfdriving car needs to be well acquainted with the geological appearance of the general area and also happenings of its surrounding. This feature of Self Driving Car enables it to take optimum decisions so that it can decide which route is best to take and can also avoid smashing into an obstacle. To achieve this, the driverless vehicles are integrated with sensor lasers, GPS mapping, advanced control systems for the elucidation of information from the sensors and a windscreen camera. This procured data is incorporated into the car's map through a simultaneous localization and mapping algorithm thus, creating a lucid image of its surroundings. These cars have bumper mounted radar and the windscreen camera which keeps track of motorists, pedestrians, road signs, street lights, traffic lights and other vehicles around the car. Also, odometer and computer vision is attached in the driverless car so that it can help to decode the information in a manner that the vehicles inbuilt software can be interpreted.

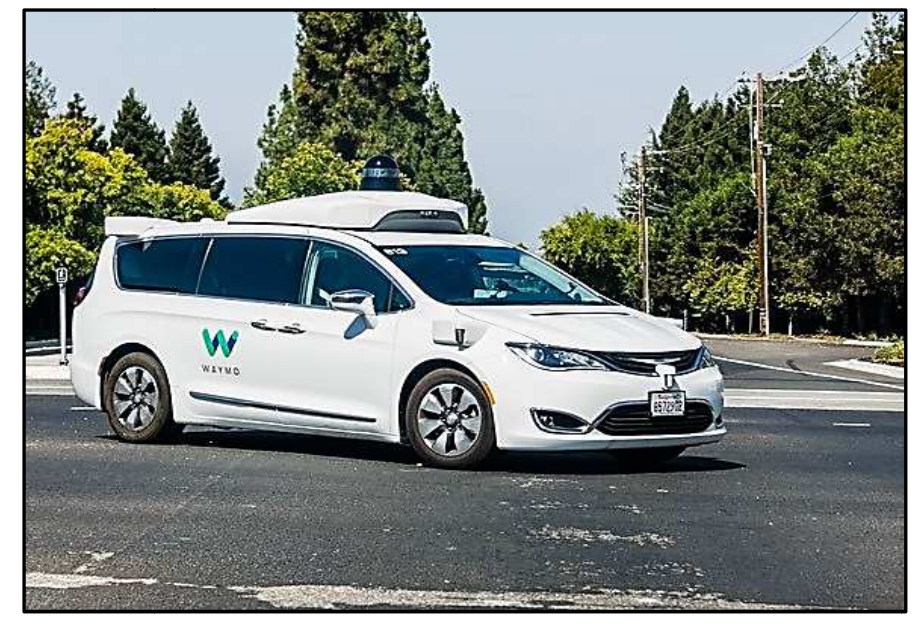

Figure 3: Self-Driving Car

For more perfect upshot, gyroscopes, tachometers and altimeters are fitted into these driverless cars. LIDAR (Lidar Detection and Range) sensors are used as they provide a meticulous and lucid image of the surroundings as compared to the GPS (Global Positioning System). To ensure that this driverless vehicle is operated similar to that operated by a professional driver, the software is programmed with neural networks that represent real driving experiences. These neurons enable the vehicle to interpret approaching bodies which help in avoiding accidents. 


\section{CLASSIFICATION OF SELF-DRIVING CAR}

As classified by the SAE International (Society of Automotive Engineers), there are different levels of automation in the driverless cars. These levels form a "Hands-On" driving experience to a "Driver-Free" mode of driving. The driver's involvement gets reduced as the level rises up.

\section{Level 0 (“ZERO Automation”)}

At this level, the vehicles are controlled by the driver. This means no braking assistance, steering controls or acceleration are required as they have no feature for self-driving. However, this may include systems that warn the driver for imminent threats but do not take any course of action.

\section{Level 1 ("DRIVER Assisted")}

Both car and driver shares responsibility in operating the car at this level. It means that either the car controls speed and the driver steers the car or vice versa. However, at this level, the car cannot handle both steering and acceleration of the vehicle. Additionally, this level may include features like lane assist, parking assistance, electronic stability controls, emergency braking and antilock braking systems.

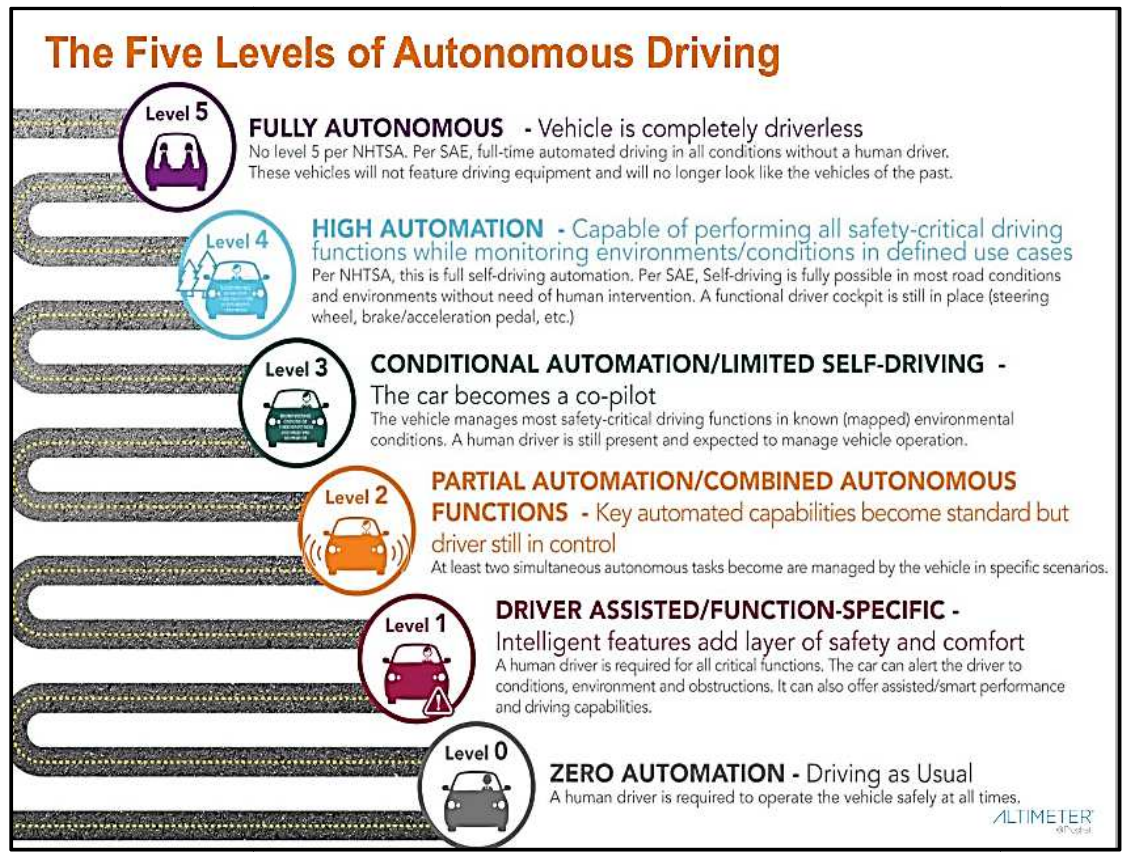

Figure 4: Levels of Autonomous Driving

\section{Level 2 ("PARTIAL Automation")}

In this, the vehicle can handle braking, acceleration and steering independently. However, the driver needs to be alert as the car does not handle real driving experiences.

\section{Level 3 ("CONDITIONAL Automation”)}

In this level, the car can handle most of the situations that may arise when manoeuvring regular traffic. However, in some cases guidance from the driver is required as there are some traffic situations which aren't programmed to handle. 


\section{Level 4 ('HIGH Automation”)}

Vehicles at this level are almost fully autonomous therefore the involvement of the driver is less. For example, when the system shows a glitch, the car reduces its speed and if it is well programmed then the vehicle is pulled off the road.

\section{Level 5 ("Fully Automation")}

In this level, the vehicles are in the top pedestal of vehicle automation. These can operate in all areas free of the driver's aid. Whether, in rough weathers or unmapped regions, the car can easily navigate without any difficulties. So far no such cars are designed.

\section{COMPONENTS OF SELF-DRIVING CAR}

\section{Video Cameras}

Small, inexpensive and highly developed cameras are used which can see colour. Multiple RGB (Red Green Blue) cameras are used for different functions. The main issue with these cameras is that they don't see vividly at night and in weather like fog, snow and hard rain.

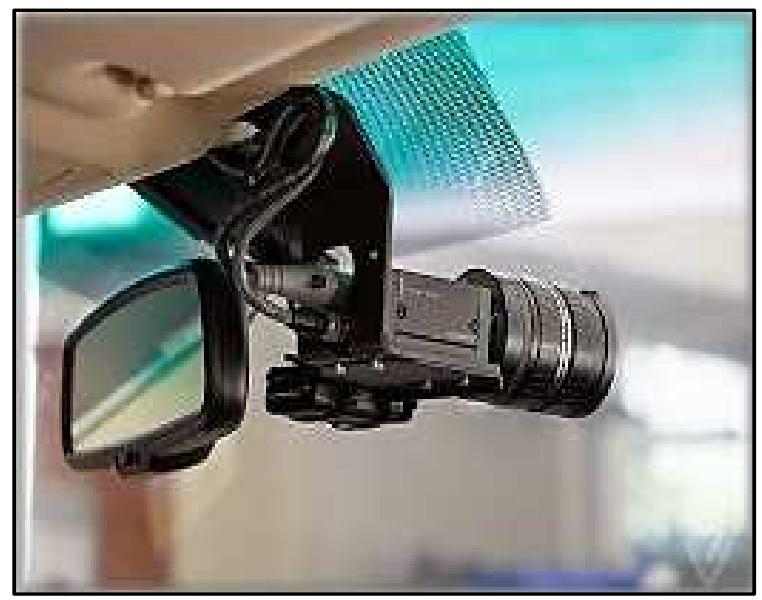

Figure 5: Video Camera

Distant objects are difficult to determine, so short range is another limitation. The complex picture captured by these cameras requires a lot of memory and special software like artificial intelligence and machine vision to interpret what they see. The New Vision System which uses two cameras in a stereo configuration overcomes some of these snags.

\section{Radar}

The alternate view of the driving surroundings is given by the Single-Chip radars which are available now. They detect objects by reflecting radio waves at a significant distance. 


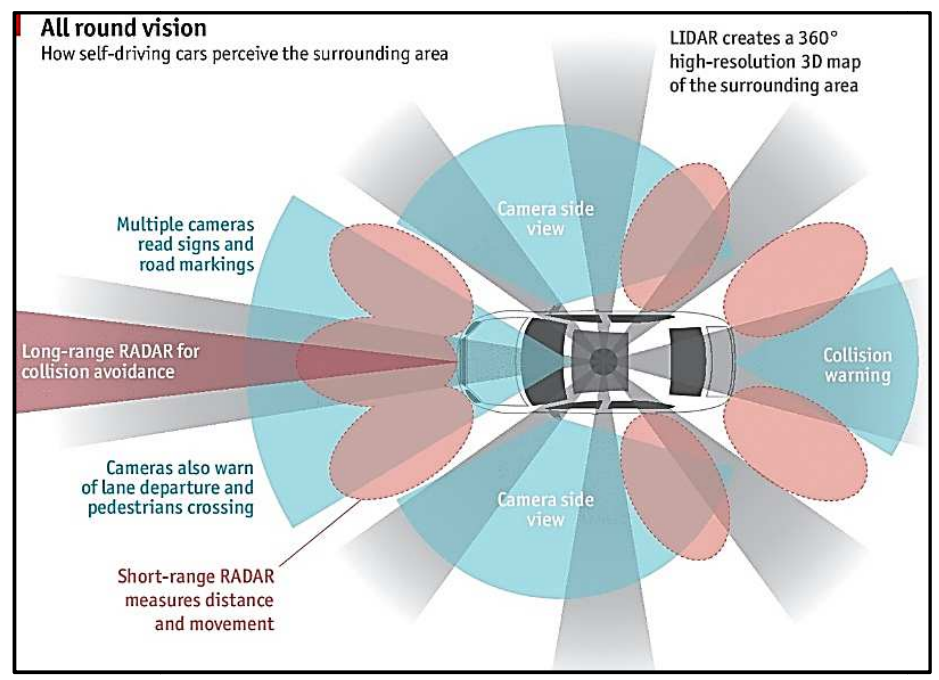

Figure 6: Radar

\section{Ultrasonic Sensor}

Totally a different view is given by these short-range sensors. These are fitted inside mirrors for close detection. It also assists in parking. These operate at a frequency of $58 \mathrm{kHz}$. These sensors are easily incorporated into the vehicle and they are inexpensive too.

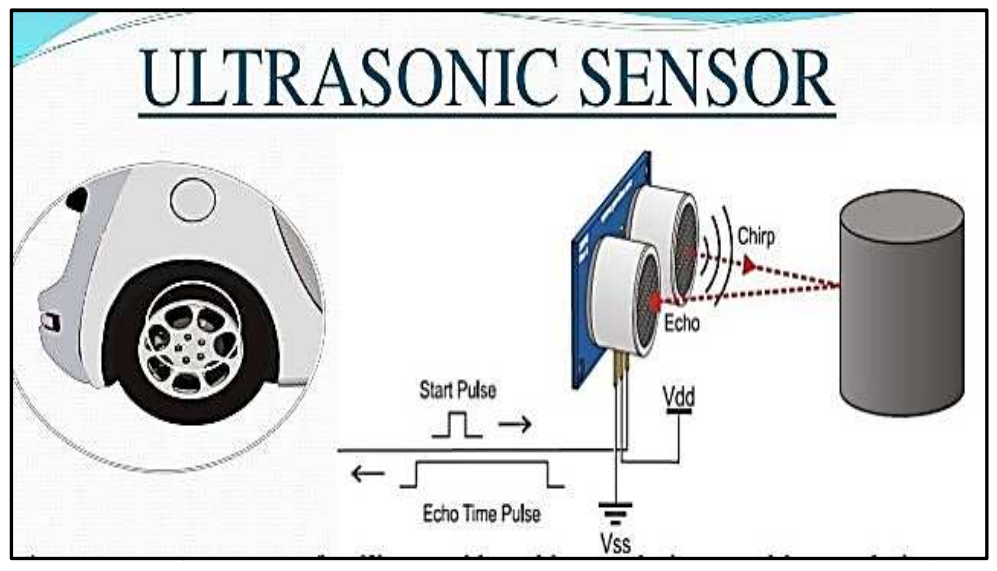

Figure 7: Ultra Sonic Sensor

\section{Software}

The software must include the various sensor inputs into a composite view of the area around the vehicle. The view is changing constantly so the software can respond to the changes quickly. It begins with an operating system likeBlackberry, QNX, Microsoft or some Linux-offshoot. Real-time processing with minimal latency is essential. The software is a mix of standard algorithms plus DSP. Artificial intelligence (AI) software using neural networks and deep machine learning do the sensor fusion computations.

\section{Processor}

There are some special processors which are needed to crunch the massive amount of data. This invites for heterogeneous processing, a group of diverse processors which includes general-purpose CPU's, Graphic Processing Units (GPU's), and DSP's from vendors like AMD and NVIDIA. Multiple vendors are acquiring processor business including 
NXP, Intel, Texas Instruments and Qualcomm. FPGA's are also being applied to get the speed which is needed for processing operations. The processors will decide what to do after analysing the inputs. Also, they will warn the driver with audible alerts. In a self-driving mode, the decisions will automatically get translated into controlling actions for braking, steering, accelerating/decelerating.

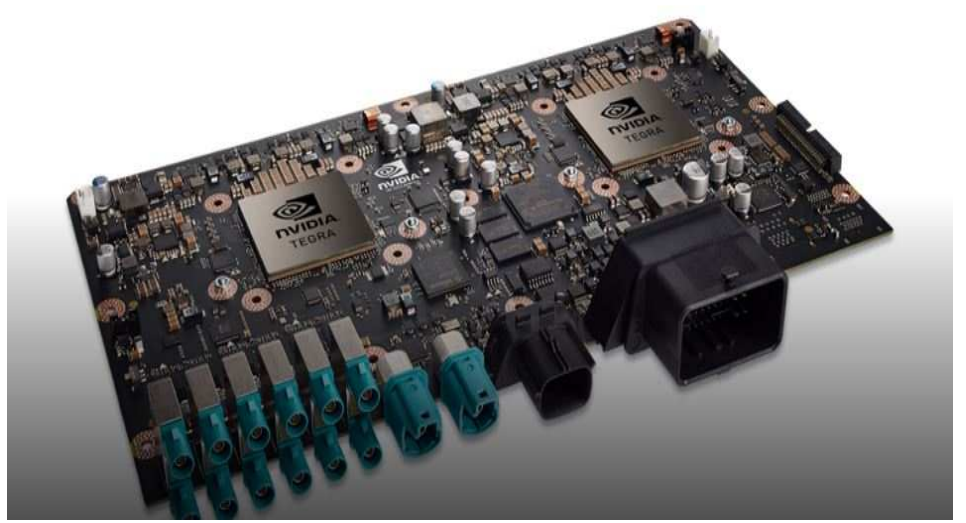

Figure 8: Processor

\section{Artificial Intelligence (AI)}

Self-Driving cars rely on Artificial Intelligence to make judgements. When the object gets identified on the street, AI determines how the car should behave in that situation. To train such intelligence, a huge amount of data is used by the scientists so that the algorithm can learn objects on the road.

\section{Mapping}

For the driverless car, a more advanced map is needed from the map which we use in our Smartphone. The sensors and cameras can detect objects on the street so it would be safer to have a precise 3D map of the route so the car would be familiar with all aspects of the street and can focus on detecting other elements. 3D maps also provide tremendous results during extreme weather conditions, when the data which is provided by the cameras and sensors are insufficient. Mapping is challenging as it requires a lot of data to produce a precise representation of the world. Even Google Map has low fidelity for rural areas.

\section{Infrastructure}

When automobile replaced horse carriages a new infrastructure was needed at that time similarly, self-driving car also requires an updated infrastructure. Also we need to build connected infrastructure as a way for driverless cars to communicate with nearby infrastructure. The infrastructure overhaul comes with a hefty price tag, but it is important for the safety of driverless cars.

\section{Lidar}

LIDAR paints the surrounding area with narrow laser beams which detect the reflections to generate a picture of the environment. It is used to detect the distance and shape of the object which is present in the surrounding. LIDAR plays an important role in ensuring a safe trip. The infrared waves sent by the LIDAR can better detect smaller objects (like rocks, or a Cyclist etc.). 


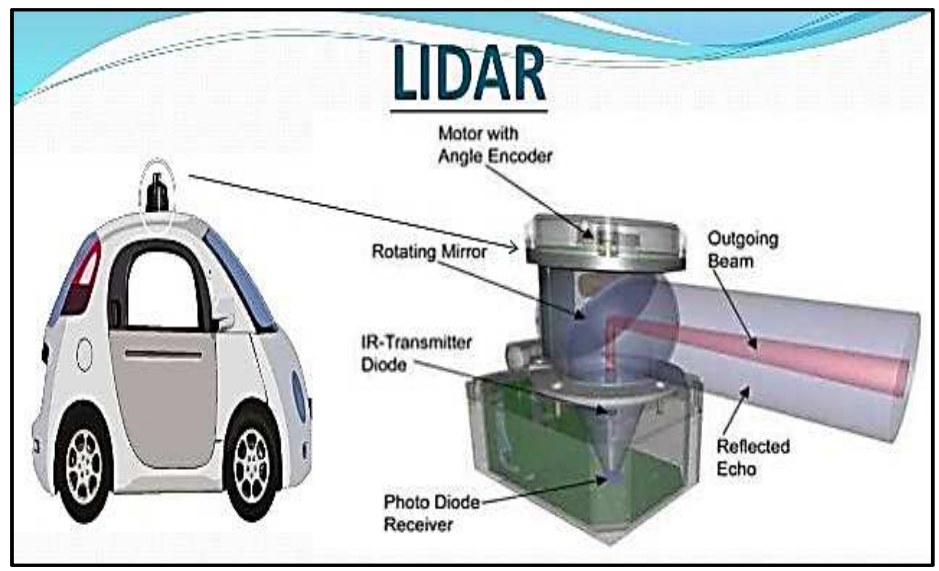

Figure 9: Lidar

By using LIDAR, a closer object can also be detected easily. The objects which are far from the vehicle are easily detected by the Radar because of its more sensitivity. The radar is explained further in this paper. LIDAR and Radar both are supportive pair for each other, they can achieve so much together for the driverless future.

\section{ADVANTAGES OF SELF-DRIVING CAR}

- Most road accidents ensue from reckless driving. Self-driving cars are expected to reduce the risk of road accidents by a considerable fraction. Self-driving vehicles will keep a distance with each other and thus it will reduce the chances of crashing.

- Self-driving cars will reduce accidents with a significant fraction, which leads to minimizing the number of insurance claims further leads to reducing the amount of money spent on accidental recovery.

- Self-driving cars are expected to reduce the traffic congestions, as each car will follow traffic rules and thus preventing the road blockages caused by driver's ignorance.

- Self-driving cars will be helpful for individuals who are physically challenged. It will minimize the struggle to locate a parking spot as your car will quickly drop you off and get a parking space independently.

\section{FUTURE SCOPE}

All necessary technologies are now beginning to become a reality. A Tech Aficionado is curious about the future of the car and also on cars swiftness and reliability. The autonomous car will be based on a number of technological modules. Some we have and some are still developing. India is not too behind in the self-driving car race, the technology is still new and budding. Also, Indian roads are a bit more challenging than the roads in western countries. The more investments done by the nation in the upcoming technologies, more incentives it will enjoy in the long run. These incentives will lead to growth in the augmentation of the job sector. Some researches have shown that the autonomous car will certainly replace ordinary cars by 2020 , but, the challenge is putting it all together in a reliable, safe and affordable way.

\section{CONCLUSIONS}

This paper discusses the basic working of sensors used in autonomous cars. Autonomous cars are developed from basic robotic cars to much efficient and practical vision guided vehicles. Also, contemporary developments in autonomous cars reflect the vivid future autonomous cars behold. Official future predictions about autonomous cars point out that most 
of the automobile companies will launch cars with semi and fully autonomous features by 2020. Most cars are expected to be fully autonomous by 2035. This paper reviewed the basic architecture of IOT and its application in autonomous vehicles.

\section{REFERENCES}

1. Muhammad A. Iqbal, OladiranG. Olaleye \& Magdy A. Bayoumi, “A Review on Internet of Things (IoT): Security and Privacy Requirements and the Solution Approaches”, Global Journal of Computer Science and Technology: E Network, Web \& Security, vol 16 Issue 7, Year 2016.

2. Kanwaldeep Kaur, Giselle Rampersad, "Trust in driverless cars: Investigating key factors influencing the adoption of driverless cars", Journal of Engineering and Technology Management, vol 48, pp 87-96, April-June 2018.

3. KeshavBimbraw, "Autonomous Cars: Past, Present and Future, A Review of the Developments in the Last Century, the Present Scenario and the Expected Future of Autonomous Vehicle Technology", Research Gate Article, January 2015.

4. Rashmi, "IoT (Internet of Things) Concept and Improved Layered Architecture”, International Journal of Engineering Development and Research, vol 6 Issue 2, pp 481-484, 2018.

5. KomalMiddha, Dr.AmandeepVerma, "Internet of Things (IOT) Architecture, Challenges, Applications: A Review", International Journal of Advanced Research in Computer Science, vol 9 No. 1, January-February 2018.

6. Sherasiya, T., Upadhyay, H., \& Patel, H. B. (2016). A survey: Intrusion detection system for internet of things. International Journal of Computer Science and Engineering (IJCSE), 5(2).

7. SomayyaMadakam, R Ramaswamy, SiddharthTripathi, “Internet of Things (IoT): A Literature Review”, Journal in Computer and Communications, pp 164-173, April 2015.

8. Muhammad Bilal, "A Review of Internet of Things Architecture, Technologies, and Analysis Smartphone-based Attacks Against 3D printers", Cornel University, June 2017.

9. Vandana Sharma, Ravi Tiwari, “A review paper on “IOT” \& It's Smart Applications", International Journal of Science, Engineering and Technology Research (IJSETR), vol 5 Issue 2, pp 472-476, February 2016.

10. FriedemannMattern, Christian Floerkemeier, "From the Internet of Computers to the Internet of Things", Distributed Systems Group, Institute for Pervasive Computing, ETH Zurich.

11. SaberTalari, MiadrezaShafie-khah, PierluigiSiano, Vincenzo Loia,

12. Aurelio Tommasetti, João P. S. Catalão, “A Review of Smart Cities Based on the Internet of Things Concept”, Energies, March 2017.

13. “Internet of Things: Science Fiction or Business Fact?”, Harvard Business Review, April 2016.

14. AbhishekBhushan, PeeyushGarg, "Internet of Things: A Review on Its Future Scope \& Challenges", National Conference on Recent Advancement in Engineering \& Science, February 2015.

15. Das, S., \& Kumar, S. Applying RCM Techniques to Household Consumer Durables through the Internet of Things.

16. M.U. Farooq, Muhammad Waseem, Muhammad Waseem, AnjumKhairi, Talha Kamal, "A Review on Internet of Things (IOT)” International Journal of Computer Applications (0975 8887), vol 113 No. 1, March 2015. 
